\title{
Conserved Quantities for Interacting Four Valent Braids in Quantum Gravity
}

\author{
Jonathan Hackett*and Yidun Wan ${ }^{\dagger}$ \\ Perimeter Institute for Theoretical Physics, \\ 31 Caroline st. N., Waterloo, Ontario N2L 2Y5, Canada, and \\ Department of Physics and Astronomy, University of Waterloo, \\ Waterloo, Ontario N2J 2W9, Canada
}

February 15, 2008

\begin{abstract}
We derive conservation laws from interactions of actively-interacting braid-like excitations of embedded framed spin networks in Quantum Gravity. Additionally we demonstrate that actively-interacting braid-like excitations interact in such a way that the product of interactions involving two actively-interacting braid-like excitations produces a resulting actively-interacting form.
\end{abstract}

*Email address: jhackett@perimeterinstitute.ca

†Email address: ywan@perimeterinstitute.ca 


\section{Contents}

1 Introduction

2 Notation 4

3 Algebra of equivalence moves: symmetries and relations 6

4 Algebra of interactions: symmetries and relations $\quad 12$

5 Conclusions $\quad 18$ 


\section{Introduction}

There has been a large amount of research effort towards a quantum theory of gravity with matter as topological invariants since a ribbonized preon model[1] was coded into local braided ribbon excitations[2]. These excitations are edges of framed three-valent spin networks present in models related to Loop Quantum Gravity with non-zero cosmological constant[3]. The topological invariants of ribbon braids are able to detect chirality and code chiral conservation laws.

The results of [2] have a serious limitation, namely that the conservation laws which preserve the excitations are exact. In other words, the braid excitations of [2] behave like solitons in integrable systems: they propagate through each other under local dynamical moves, leaving no possibility for interaction, e.g. creation and annihilation, to occur[4]. Consequently, interpreting braid excitations found in [2] as particles - in particular the Standard Model particles - is not going to work out unless interactions are successfully introduced to that model.

Although there has been a search for a modification of the dynamics studied in [2] which would allow both propagation and interactions of the local braid excitations, a new model has also been put forward, which solves the problem of interaction and opens new interesting directions worth of investigation [5, 6].

The new model extends the graphs from three-valent to four-valent spin networks, embedded in a topological three-manifold, which also gives rise to local braid excitations, and bases the dynamics on the dual Pachner moves naturally associated with four-valent graphs. The two main reasons of this extension are: that four-valent graphs and the corresponding dual Pachner moves naturally occur in spin foam models[7], and that vertices of four-valent spin networks have true correspondence to three-dimensional space. Nevertheless, a Pachner move in the model is allowed on a subgraph only when the interior of the dual topology of the subgraph is homeomorphic to an open trivial ball in $\mathbb{R}^{3}$. This condition ensures the stability of certain braid excitations [6].

This is the third of a series of papers along the research on the new model. Our previous work formulates a clear and useful graphic calculus as the tool to study the new model. We summarize our previous main results as follows. In [5] we found that 3strand braids, each of which consists of two adjacent nodes sharing three edges, are of most interest and defined equivalence moves which relate projections of diffeomorphic embeddings of the same spin network. Furthermore, we classified 3-strand braids into two primary categories: reducible braids and irreducible braids. The former contains braids which are equivalent to braids with less number of crossings under equivalence moves. [6] shows that some stable braids propagate, in the sense that under the local dynamical moves they exchange places with their adjacent substructures in the graph. This propagation is in most cases chiral, in which a braid can only propagates along an edge in the larger graph only to the left and its mirror image (which is a distinct state) can only propagates to the right. The chirality of this propagation is related to our classification of braids in [5]. Additionally, two neighboring braids can interact, in the sense 
that they merge into a new braid under local equivalence and dynamical moves; however, for this to happen either or both of them must be in a small class of braids called actively-interacting. Lastly we found that any actively-interacting braid is equivalent to some trivial braid with twists.

Due to the key role the actively-interacting braids play in this model, in this paper we study their interactions in more details in an algebraic way. Despite the power of the previously developed graphic calculus in [5, 6], a symbolic algebra would provide a concise tool, convenient for computation and more importantly more able to demonstrate conserved quantities as "quantum numbers". As the ultimate goal of this model is to interpret certain braid excitations as matter degrees of freedom in a physical way particularly to see if the braid excitations can be mapped to Standard Model particles - we need a symbolic algebra which exhibits possible symmetries in a more transparent and lucid way. Indeed, as a follow-up work of [2], in [8] similar algebraic approach is being taken for three-valent spin networks, in which each ribbon braid is only characterized by three integers: the so-called Louis numbers, named after Louis Kauffman. However, in the 4-valent case a braid is characterized by more integral quantities, as will be seen.

To these ends within this paper, we shall develop a concise algebraic notation of braids which interact actively. Within this notation, the algebraic equivalence moves are defined, and the quantities conserved under these are identified. Finally, the algebra of interactions of actively-interacting braids is developed in detail. From this the following results are found:

1. Conserved quantities exist under interactions and we are able to show the form of these conservation laws.

2. The interaction of two actively-interacting braids results in an actively-interacting braid.

It is worth noting that the algebraic notation proposed in this paper will be used in the parallel papers [9, 10, 11]. [9] extends the algebraic notation and conserved quantities of actively-interacting braids to all stable braids. Based on this, [10] discovers the discrete transformations of braids, which are mapped to C, P, T, and their combinations. Then [11] proposes an effective theory of our braid excitations in terms of Feynman diagrams, which implies the analogy between actively-interacting braids and bosons. This implication conversely motivate this paper as well.

\section{Notation}

We will develop and use an algebraic notation, with, however, keeping a graphic notation wherever necessary for an illustrative purpose. We adopt the graphical notation we proposed in [5, 6]. Fig. 1] shows an example of a 3-strand braid in this notation. More precisely, Fig. 1 is a braid diagram which is a projection of the true 3-strand braid embedded 
in a topological three manifold. Each spin network can be embedded in various ways, some of which are diffeomorphic to each other. The projection of a specific embedding of a braid is called a braid diagram; many braid diagrams are equivalent and belong to the same equivalence class, in the sense that they correspond to the same braid and can be transformed into each other by equivalence moves[5]. Thus, a braid refers to the whole equivalence class of its braid diagrams. Nevertheless, one can choose a braid diagram of an equivalence class as the representative of the class, we therefore will not distinguish a braid from a braid diagram in the sequel unless an ambiguity arises. Besides, a braid always means a 3-strand braid.

Among all the stable braids, a small class is called actively-interacting, in the sense that they interact onto any other braid as long as the interaction condition is met. These braids are indeed "active" because they also propagate both to its left and right[6], so they are able to find other braids to interact. The interactions of passive braids, i.e. braids which are not actively-interacting, are mediated by actively-interacting braids, which implies the possibility that actively-interacting braids behave bosonic[11]. According to [6, 5], all actively interacting braids happen to be both completely left- and rightreducible, i.e. such a braid can always be represented by a trivial braid diagram with possibly twists on its three strands and two external edges. This fact largely reduces the complexity of actively-interacting braids because a trivial braid is fully characterized by its twists and end-nodes' states, no crossings to be worried about. Fig. 2(a) depicts a trivial braid in general.

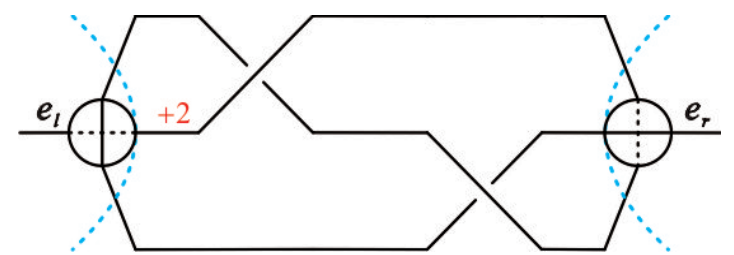

Figure 1: A typical 3-strand braid diagram formed by the three common edges of two end-nodes. The region between the two dashed line satisfies the definition of an ordinary braid. Edges $e_{l}$ and $e_{r}$ are called external edges. There is also a right handed twist of 2 units on the middle strand. In this figure the left end-node is in an ' + ' state while the right end-node is in an '-' state.

Therefore, in this paper we concentrate on trivial braid diagrams that represent activelyinteracting braids. To study these braids in more details and in a more efficient way, we need a nice algebraic notation. We found that it is handy to denote a generic trivial braid shown in Fig. 2(a) algebraically by the notation in Eq. 1.

$$
{ }_{T_{l}}^{S_{l}}\left[T_{a}, T_{b}, T_{c}\right]_{T_{r}}^{S_{r}},
$$

where $S_{l}, S_{r}$, being ' + or '-', are respectively the states of the left and right end-nodes of a braid, $T_{l}, T_{r}$, called the left and right external twists, are respectively the twists on 
the left and right external edges of a braid, and the triple $\left[T_{a}, T_{b}, T_{c}\right]$ records the twists on the three strands respectively in the order shown in Fig. 2(a), which are thus named the internal twists. The subscript $a$ of an internal twist $T_{a}$ is abstract and has no meaning before its position in the triple is fixed. So $\left[T_{a}, T_{b}, T_{c}\right]=\left[T_{d}, T_{e}, T_{f}\right]$ means respectively $T_{a}=T_{d}, T_{b}=T_{e}$, and $T_{c}=T_{f}$. In the rest of the paper, we will also consider the addition of two triple of twists, i.e. $\left[T_{a}, T_{b}, T_{c}\right]+\left[T_{d}, T_{e}, T_{f}\right]=\left[T_{a}+T_{d}, T_{b}+T_{e}, T_{c}+T_{f}\right]$.

All twists are valued in $\mathbb{Z}$ in units of $\pi / 3[5]$. For example, the braid in Fig. $2(b)$ is denoted in the algebraic notation by ${ }_{-1}^{+}[-1,1,+2]_{0}^{-}$. Note that for a trivial braid diagram that interact actively, the set $\left\{T_{l}, S_{l}, T_{a}, T_{b}, T_{c}, S_{r}, T_{r}\right\}$ characterizing it is not completely arbitrary but rather has the following constraints.

1. $S_{l} \equiv S_{r}$. If a braid is actively-interacting, each of its trivial braid diagrams must have both end-nodes in the same state (so the braid in Fig. 2(b) does not actively interact).

2. the triple $\left[T_{a}, T_{b}, T_{c}\right]$ is not arbitrary; however, the general pattern of them, ensuring active interaction, has not yet been found. Nevertheless, the algebra formulated in this paper may turn out to be helpful to resolve this problem.

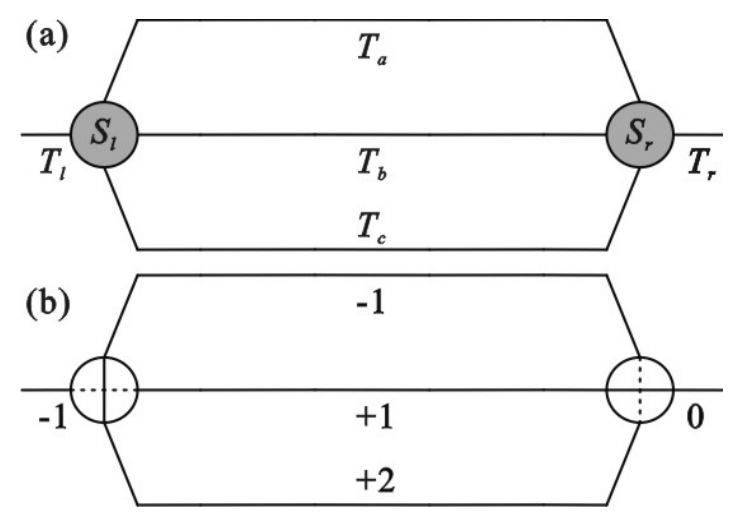

Figure 2: (a) is a trivial braid in general, in which the two nodes are filled in grey, indicating arbitrary states. (b) is a concrete example of (a).

\section{Algebra of equivalence moves: symmetries and relations}

In the first of this series of papers[5], we discovered equivalence moves on embedded 4-valent spin networks by which braid diagrams are classified. Any braid diagram in an equivalence class can be chosen to be the representative; however, for the purpose of studying braid propagation and interaction, we found that each equivalence class of braid diagrams has a unique representative which has two twist-free external edges. If 
the unique representative of a braid diagram interacts actively, so does the whole class. Nevertheless, as mentioned above, in this paper we are mainly interested in actively interacting braids which have trivial braid diagrams; hence, it is now more convenient to represent an equivalence class of braid diagrams by trivial braid diagrams in the class which are certainly not unique. In other words, if a trivial braid in our algebraic notation corresponds to a particle, there are many other equivalent ones corresponding to the same particle. This fundamental degeneracy of a particle is a direct result of diffeomorphism invariance because equivalence moves do not change the diffeomorphism of an embedding. Therefore, it is necessary to find out for any equivalence class of braid diagrams, in an algebraic way adapted to our new algebraic notation, how a trivial representative is related to another in the same class, in particular how the set of quantities characterizing the representative changes, and more importantly what the conserved quantities are.

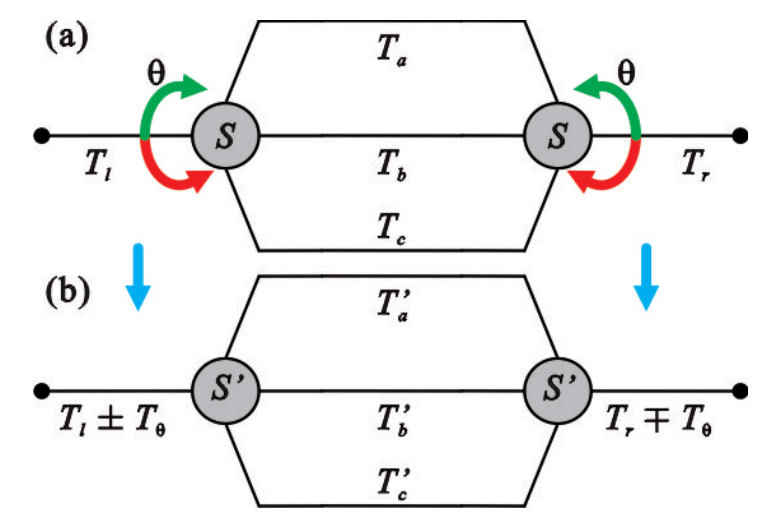

Figure 3: (a) is a actively interacting trivial braid in general; (b) is obtained from (a) by same amount of rotations respectively on both end-nodes of (a) in the same direction (either green or red) shown in the figure. $T_{\theta} \in \mathbb{Z}$ is the twist induced by the rotation $\theta$. $\left[T_{a}^{\prime}, T_{b}^{\prime}, T_{c}^{\prime}\right]=\left[P_{\theta}^{S}\left(T_{a}, T_{b}, T_{c}\right)\right]$, where $P_{\theta}^{S}$ is the permutation depending on both $S$ and $\theta$.

The tools we can utilize have already been introduced in [5] in detail, namely the rotations. That is, we can use, e.g. $\pi / 3$ rotations, to take a trivial braid to another. Concentrating on trivial braids only, however, we do not expect any crossing to appear because a single rotation on an end-node of a braid diagram creates/annihilates crossings, so we must apply rotations on both end-nodes of a trivial braid simultaneously, such that no crossing arises. The idea is illustrated in Fig. 3. A remark is that the "same direction" we mentioned in the caption of Fig. 3 is with respect to the surface the braid is projected on, i.e. this piece of paper; nonetheless, in our definition of rotation with respect to the rotation axis, i.e. the external edges, the rotation $\theta$ of the left end-node and the one of the right end-node are of opposite handedness. This has two immediate consequences: 1)in Fig. 3(b), while the resulted twist on the left external edge is $T_{l} \pm T_{\theta}$, the one on the right is $T_{r} \mp T_{\theta}$, a sign difference appears; and 2)this process creates no crossing and interestingly no extra twists on the three strands of the braid but rather results in the twists $\left[T_{a}^{\prime}, T_{b}^{\prime}, T_{c}^{\prime}\right]$ 
in Fig. 3(b) as a permutation $\left[P_{\theta}^{S}\left(T_{a}, T_{b}, T_{c}\right)\right]$ of the three twists in Fig. 3(a). Note that $P_{\theta}^{S}$ depends also on $S$, the state of the end-nodes before being rotated, which is a special property of rotations in 4-valent case. We name a whole procedure of this type on a braid a simultaneous rotation.

We can describe the action of a simultaneous rotation on a trivial braid algebraically. We first define an operator for a simultaneous rotation, which acts simultaneously on both end-nodes of braid, by $R_{n,-n}$, where $n$ is the amount of rotation valued in units of $\pi / 3$, i.e. $n \in \mathbb{Z}$, and the signs, which must be opposite in the first and the second subscripts, indicate respectively the handedness of rotations on left and right end-nodes. The second index of $R$ seems a bit redundant; however, we keep it to make the rotation handedness explicit. Moreover, a state $S$ is simply a sign, + or - , so we let $-(+)=-$ and $-(-)=+$, i.e. $-S=\bar{S}$ both denoting the opposite of $S$. Hence, in general we have

$$
R_{n,-n}\left({ }_{T_{l}}^{S}\left[T_{a}, T_{b}, T_{c}\right]_{T_{r}}^{S}\right)={ }_{T_{l}+n}^{(-)^{n} S}\left[P_{n}^{S}\left(T_{a}, T_{b}, T_{c}\right)\right]_{T_{r}-n}^{(-)^{n} S}
$$

where $(-)^{n}$ enters the equation because a $\pi / 3$ rotation changes the end-node state once. When $n$ is even, $R_{n,-n}$ does not change the end-node state of a braid, it is then christened an even simultaneous rotation, otherwise it is named an odd simultaneous rotation. The RHS of Eq. 2 clearly presents the three effect of a simultaneous rotation $R_{n,-n}$ has three effects on a trivial braid: the change of end-node state depending on $n$, the change of external twists depending on $n$, and the induced permutation $P_{n}^{S}$ on the triple of internal twists, which is determined by the end-node state $S$ before the action of $R, n$, and the handedness of the rotation on the left end-node.

In view of that all possible rotations on a node of an embedded 4-valent spin network are generated by $\pi / 3$ rotations [5], which correspond to $n= \pm 1, R$ satisfies

$$
R_{n,-n}=R_{1,-1}^{(n)},
$$

where $(n)$ means $n$-th power, e.g. $R_{1,-1}^{(2)}=R_{2,-2}$ and $R_{1,-1}^{(-2)}=R_{-2,2}$. However, Eq. 3 is actually formal and is not as simple as it appears to be. The reason lies in the fact that a simultaneous rotation induces a permutation depending on the end-node state before taking the rotation, and that a $\pi / 3$ inverses the end-node state. More precisely, for example, a rotation $R_{2,-2}$ is equal to two $R_{1,-1}$ in a row; the first $R_{1,-1}$ induces a permutation, flipping the end-node state from, say $S$, to $\bar{S}$, so the permutation induced by the second $R_{1,-1}$ now actually respects $\bar{S}$ rather than $S$. This reads, mathematically,

$$
R_{2,-2}\left({ }_{T_{l}}^{S}\left[T_{a}, T_{b}, T_{c}\right]_{T_{r}}^{S}\right)=R_{1,-1}\left({ }_{T_{l}+1}{ }^{\bar{S}}\left[P_{1}^{S}\left(T_{a}, T_{b}, T_{c}\right)\right]_{T_{r}-1}^{\bar{S}}\right)={ }_{T_{l}+2}^{S}\left[P_{1}^{\bar{S}} P_{1}^{S}\left(T_{a}, T_{b}, T_{c}\right)\right]_{T_{r}-2}^{S}
$$

In other words, the permutation induced by $R_{2,-2}$ is $P_{1}^{\bar{S}} P_{1}^{S}$ but not $P_{1}^{S} P_{1}^{S}$, we'll call this permutation $P_{2}^{S}$. This is readily generalized to higher rotations. Clearly, an induced permutation is an element of the permutation group $S_{3}=\operatorname{Sym}\left(\left[T_{a}, T_{b}, T_{c}\right]\right)$. In terms of disjoint cycles,

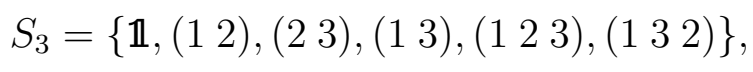


where for example, (1 2) means exchanging elements 1 an 2 in the triple $\left[T_{a}, T_{b}, T_{c}\right]$ and

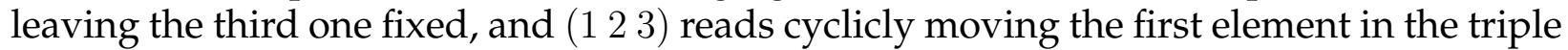
to the second, the second to the third, and the third to the first. Moreover, for a product of permutations, the order of its action on a triple is, in our convention, from right to left.

It is then demanded but sufficient to study a simultaneous rotation of $\pi / 3$ on both endnodes of a trivial braid to fully understand how a general simultaneous rotation works and to obtain our desired algebraic relations in general. This is done in Fig. 4 .
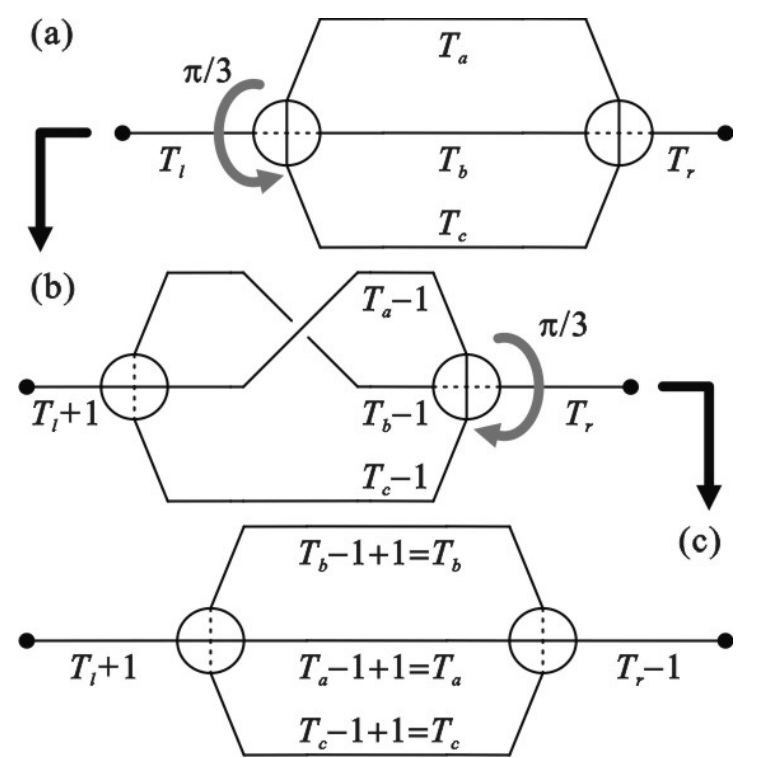

Figure 4: A simultaneous rotation of $\pi / 3$ is split into two steps, namely two $\pi / 3$ rotations respectively on the two end-nodes of the braid in (a). After a right-handed $\pi / 3$ rotation on the left end-node, (a) gives rises to (b), in which all twists but $T_{r}$ are modified from those in (a) accordingly, and a crossing is created. However, by another left-handed $\pi / 3$ rotation on the right end-node of (b), a trivial braid appears again in (c). One sees that the induced permutation by this simultaneous rotation is $P_{+1}^{+}=\left(\begin{array}{ll}1 & 2\end{array}\right)$.

To obtain the exact algebraic form of this simultaneous rotation of $\pi / 3$, we can split the rotation into two rotations respectively on the two end-nodes of the braid in Fig. 4. Our choice is to first rotate left and then the right end-nodes of the trivial braid in (a); it is obvious that the order of this splitting does not matter, which guarantees that a simultaneous rotation is well-defined. This rotation gives an equivalence relation between the trivial braid in Fig. 4(a) and the one in (c), whose algebraic form is now clear:

$$
R_{+1,-1}\left({ }_{T_{l}}^{+}\left[T_{a}, T_{b}, T_{c}\right]_{T_{r}}^{+}\right)={ }_{T_{l}+1}^{-}\left[T_{b}, T_{a}, T_{c}\right]_{T_{r}-1}^{-} .
$$

The permutation implied in this relation is $\left[P_{+1}^{+}\left(T_{a}, T_{b}, T_{c}\right)\right]=\left[T_{b}, T_{a}, T_{c}\right]$, i.e. $P_{+1}^{+}=\left(\begin{array}{ll}1 & 2\end{array}\right)$. Since each node has only two states and each rotation has only two directions, it is not 
hard to enumerate all possible simultaneous $\pi / 3$ rotations on all possible actively interacting trivial braids, in a way similar to what we do in Fig. 44. For future convenience, we list all such relations in the following table.

$$
\begin{aligned}
& R_{+1,-1}\left(T_{T_{l}}^{+}\left[T_{a}, T_{b}, T_{c}\right]_{T_{r}}^{+}\right)={ }_{T_{l}+1}^{-}\left[T_{b}, T_{a}, T_{c}\right]_{T_{r}-1}^{-} \quad P_{+1}^{+}=\left(\begin{array}{ll}
12 \\
T_{-1,+}
\end{array}\right. \\
& R_{-1,+1}\left({ }_{T_{l}}^{+}\left[T_{a}, T_{b}, T_{c}\right]_{T_{r}}^{+}\right)={ }_{T_{l}-1}^{-}\left[T_{a}, T_{c}, T_{b}\right]_{T_{r}+1}^{-} \quad P_{-1}^{+}=\left(\begin{array}{ll}
23 \\
R_{+}
\end{array}\right) \\
& R_{+1,-1}\left({ }_{T_{l}}^{-}\left[T_{a}, T_{b}, T_{c}\right]_{T_{r}}^{-}\right)={ }_{T_{l}+1}^{+}\left[T_{a}, T_{c}, T_{b}\right]_{T_{r}-1}^{+} \quad P_{+1}^{-}=\left(\begin{array}{ll}
2 & 3
\end{array}\right) \\
& R_{-1,+1}\left({ }_{T_{l}}^{-}\left[T_{a}, T_{b}, T_{c}\right]_{T_{r}}^{-}\right)={ }_{T_{l}-1}^{+}\left[T_{b}, T_{a}, T_{c}\right]_{T_{r}+1}^{+} \quad P_{-1}^{-}=(12)
\end{aligned}
$$

Table 1: All possible simultaneous $\pi / 3$ rotations on trivial braids which interact actively.

From Table 1, one can see that the following quantities are invariant under these simultaneous rotations:

1. $T_{l}+T_{r} ;$

2. $T_{a}, T_{b}$, and $T_{c}$ are conserved individually modulo permutation;

3. $S^{2}$ (which additionally means that the interacting nature of braids is preserved under the simultaneous rotations).

Furthermore, because of Eq. 3, this result applies to any simultaneous rotation. There are also two derived conserved quantities: specifically $T_{a}+T_{b}+T_{c}$ and $T_{\text {total }}=T_{l}+$ $T_{a}+T_{b}+T_{c}+T_{r}$. $T_{\text {total }}$ is consistent to the overall conserved quantity found in [5]: the sum of all twists and crossings of a subgraph - in particular a braid diagram - under any equivalent move. For a trivial braid no crossing exists and so the overall conserved quantity consists of twists only, this is $T_{\text {total }}$. All these conserved quantities are invariants under diffeomorphic embeddings.

Relations in Table 1 are generating relations for all possible simultaneous rotations, for example,

$$
\begin{aligned}
R_{+3,-3}\left({\stackrel{+}{T_{l}}}_{l}^{+}\left[T_{a}, T_{b}, T_{c}\right]_{T_{r}}^{+}\right) & =R_{+2,-2}\left({ }_{T_{l}+1}^{-}\left[T_{b}, T_{a}, T_{c}\right]_{T_{r}-1}^{-}\right) \\
& =R_{+1,-1}\left({ }_{T_{l}+2}^{+}\left[T_{b}, T_{c}, T_{a}\right]_{T_{r}-2}^{+}\right) \\
& ={ }_{T_{l}+3}^{-}\left[T_{c}, T_{b}, T_{a}\right]_{T_{r}-3}^{-},
\end{aligned}
$$

where the $\pi$ rotation is realized by three consecutive $\pi / 3$ rotations. It is easy to check by our graphic calculus that the above calculation is indeed correct. Note that one must be careful of performing an induced permutation at each step in such a calculation as permutation depends on both the end-node state before the rotation and the rotation handedness. In particular we will see three important identities regarding the induced permutations shortly. One can observe from Table 1 that

$$
\begin{aligned}
& P_{ \pm 1}^{S} P_{\mp 1}^{\bar{S}}=\mathbb{1} \\
& P_{ \pm 1}^{S}=P_{\mp 1}^{\bar{S}},
\end{aligned}
$$


where $S$ is the end-node state and $\bar{S}$ is its opposite. This can actually be generalized to the following lemma:

\section{Lemma 1.}

$$
\begin{aligned}
P_{2 n}^{S} P_{-2 n}^{S} & \equiv \mathbb{1} \\
P_{2 n+1}^{S} P_{-(2 n+1)}^{\bar{S}} & \equiv \mathbb{1} \\
P_{n}^{S} & \equiv P_{-n}^{\bar{S}},
\end{aligned}
$$

where $n \in \mathbb{Z}$.

Proof. We shall begin by proving Eq. 5 (Eq. 6 follows similarly). First we have

$$
P_{ \pm 2}^{S} P_{\mp 2}^{S}=P_{ \pm 1}^{\bar{S}} P_{ \pm 1}^{S} P_{\mp 1}^{\bar{S}} P_{\mp 1}^{S}=P_{ \pm 1}^{\bar{S}} \mathbb{1} P_{\mp 1}^{S}=\mathbb{1},
$$

where the last two equalities hold by repeatedly applying Eq. 4 , and $\bar{S}$ appears as a single $\pi / 3$ rotation flips $S$. Using this we obtain

$$
\begin{aligned}
P_{ \pm 2 n}^{S} P_{\mp 2 n}^{S} & =\left(P_{ \pm 2}^{S}\right)^{n}\left(P_{\mp 2}^{S}\right)^{n} \\
& =\left(P_{ \pm 2}^{S}\right)^{n-1} \underbrace{P_{ \pm 2}^{S} P_{\mp 2}^{S}}_{=\mathbb{1}}\left(P_{\mp 2}^{S}\right)^{n-1} \\
& =\left(P_{ \pm 2}^{S}\right)^{n-1}\left(P_{\mp 2}^{S}\right)^{n-1} \\
& =\mathbb{1},
\end{aligned}
$$

where the last line results from repeatedly performing the same expansion as in the second line. We then prove Eq. 7 by induction. It is already true for $n= \pm 1$ (Eq. 4). Assume it holds for $-(k-1) \leq n \leq k-1$ for some $k \in \mathbb{N}$, then we have for $|n|=k$

$$
P_{ \pm k}^{S}= \begin{cases}P_{ \pm 1}^{\bar{S}} P_{ \pm(k-1)}^{S}=P_{\mp 1}^{S} P_{\mp(k-1)}^{\bar{S}}=P_{\mp k}^{\bar{S}} & \text { if } k \text { is even, } \\ P_{ \pm 1}^{S} P_{ \pm(k-1)}^{S}=P_{\mp 1}^{\bar{S}} P_{\mp(k-1)}^{\bar{S}}=P_{\mp k}^{\bar{S}} & \text { if } k \text { is odd. }\end{cases}
$$

Thus Eq. 7 holds. The equations below are easy to derive; they are listed here for possible future use.

$$
\begin{aligned}
P_{2}^{+}=P_{-2}^{-} & =\left(\begin{array}{lll}
1 & 3 & 2
\end{array}\right) \\
P_{-2}^{+}=P_{2}^{-} & =\left(\begin{array}{lll}
1 & 2 & 3
\end{array}\right) \\
P_{6 n+3}^{ \pm} & \equiv\left(\begin{array}{lll}
1 & 3
\end{array}\right) \\
P_{6 n}^{ \pm} & \equiv \mathbb{1},
\end{aligned}
$$

where $n \in \mathbb{Z}$. 


\section{Algebra of interactions: symmetries and relations}

Now that we consider trivial braids which interact actively, we may ask a question: do two actively interacting braids always interact to form another actively interacting braid? To answer this question, we need to first find out if two adjacent actively interacting braids, say $B$ and $B^{\prime}$, always interact. One may feel this question somehow bizarre at first glance because how it is possible that two actively interacting braids do not interact.
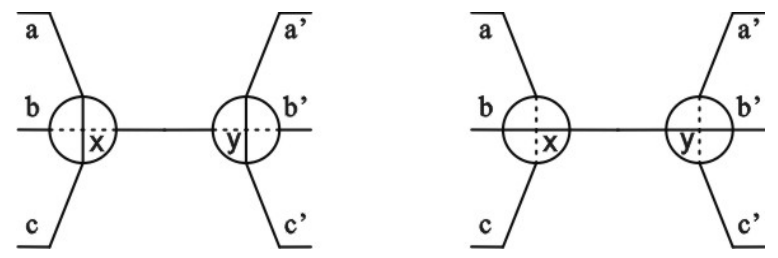

Figure 5: The only two possible configurations allowing a $2 \rightarrow 3$ move.

Nevertheless, the reason to ask such a question is two-fold. Firstly, the first step of performing an interaction of $B$ and $B^{\prime}$ is doing a $2 \rightarrow 3$ move about the two adjacent end-nodes of $B$ and $B^{\prime}$ respectively; however, a $2 \rightarrow 3$ move is doable only if the two adjacent nodes are of the same state and their common edge is twist-free[6], as shown in Fig. 5. Secondly, in our previous work, we chose to represent an equivalence class of braid diagrams by its unique representative which has twist-free external edges but in this paper we represent a class by its trivial braid diagrams whose external edges are not necessary twist-free. Therefore, when two trivial braids $B$ and $B^{\prime}$ meet each other, to see if they interact we should first check if we can put their adjacent end-nodes in the same state and with a twist-free common edge in between, which constructs the interaction condition. In search of the answer to this question, an algebra of interactions between actively-interacting braids will be naturally developed.

For two actively-interacting trivial braids $B$ and $B^{\prime}$ with $B$ on the left of $B^{\prime}$, there are two general cases to study, which are illustrated in Fig. 6. In Fig. 6(a), the right end-node of $B$ and the left end-node of $B^{\prime}$ are already in the same state; hence, the task is not only to get rid of the overall twist $T_{r}+T_{l}^{\prime}$ on the common edge but also to keep the adjacent end-nodes in the same state. We know that only multiples of $2 \pi / 3$ rotations do not change the state of the node being rotated, we thus demand that in this case $T_{r}+T_{l}^{\prime}=2 n, n \in \mathbb{Z}$. For example, if $T_{r}+T_{l}^{\prime}=2$, we can perform a left-handed $2 \pi / 3$ rotation either on the right end-node of $B$ or on the left end-node of $B^{\prime}$, such that the two adjacent end-nodes are still in state $S$ and their common edge is now twist-free.

As to Fig. 6(b), the right end-node of $B$ and the left end-node of $B^{\prime}$ are in opposite states, so we should rotate either of them to set them in the same state and annihilate the twist $T_{r}+T_{l}^{\prime}$ at the mean time. Based on the fact that only odd multiples of $\pi / 3$ rotations flip the state of the node under rotation, we clearly must have in this case, $T_{r}+T_{l}^{\prime}=$ $2 n+1, n \in \mathbb{Z}$. 


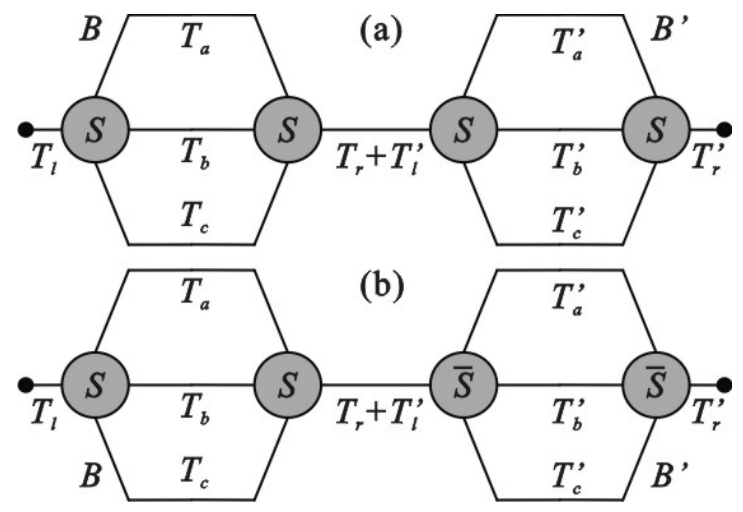

Figure 6: Two general cases in which two actively interacting braids $B$ and $B^{\prime}$ meet each other: (a) $B$ and $B^{\prime}$ have adjacent end-nodes in the same state; and (b) $B$ and $B^{\prime}$ have adjacent end-nodes in opposite states. $\bar{S}$ stands for the opposite of $S$. The interaction condition in cases (a) and (b) are respectively $T_{r}+T_{l}^{\prime}=2 n$ and $T_{r}+T_{l}^{\prime}=2 n+1, n \in \mathbb{Z}$.

There is a good news. As long as the above interaction condition holds, such that a $2 \rightarrow 3$ move can be done on the two neighboring end-nodes of $B$ and $B^{\prime}$, according to our definitions of interaction and of actively-interacting braids, $B$ and $B^{\prime}$ can definitely interact to form another braid, written as $B+B^{\prime}$, which is also actively interacting. The reason is understood this way: the interaction, left or right, of $B+B^{\prime}$ onto an arbitrary braid (interacting or not), say $B^{\prime \prime}$, can always be thought as a composite process of two interactions, namely $\left(B+B^{\prime}\right)+B^{\prime \prime}=B+\left(B^{\prime}+B^{\prime \prime}\right)$, in which $B$ is the active braid in the second step, or $B^{\prime \prime}+\left(B+B^{\prime}\right)=\left(B^{\prime \prime}+B\right)+B^{\prime}$, in which $B^{\prime}$ is the active one in the second step. In other words, an interaction is associative, with however, an active braid always playing the active role.

Nevertheless, how does $B+B^{\prime}$ look like as represented by a trivial braid diagram? To perform the interaction, either $B^{\prime}$ s right end-node or $B^{\prime \prime}$ s left end-node may be rotated before one can do a $2 \rightarrow 3$ move, which disguises the form of $B+B^{\prime}$ from being guessed directly. Fortunately, this issue can be tackled as follows.

Let us study the case in Fig. 6(a). Supposing the condition, $T_{r}+T_{l}^{\prime}=2 n$, holds, we have two subcases. The first is the significantly easier to prove and so we shall address it first:

Lemma 2. Given two actively interacting braids $B={ }_{T_{l}}^{S}\left[T_{a}, T_{b}, T_{c}\right]_{T_{r}}^{S}$ and $B^{\prime}={ }_{T_{l}^{\prime}}^{ \pm S}\left[T_{a}^{\prime}, T_{b}^{\prime}, T_{c}^{\prime}\right]_{T_{r}^{\prime}}^{ \pm S}$ satisfying the interaction conditions with $T_{r}+T_{l}^{\prime}=0$, the interaction of $B$ and $B^{\prime}$ gives $B^{f}=$ ${ }_{T_{l}}^{S}\left[\left(T_{a}, T_{b}, T_{c}\right)+\left(T_{a}^{\prime}, T_{b}^{\prime}, T_{c}^{\prime}\right)\right]_{T_{r}^{\prime}}^{S}={ }_{T_{l}}^{S}\left[T_{a}+T_{a}^{\prime}, T_{b}+T_{b}^{\prime}, T_{c}+T_{c}^{\prime}\right]_{T_{r}^{\prime}}^{S}$

Proof. As $T_{r}+T_{l}^{\prime}=0$, no rotation is needed; hence, according to [6], $B+B^{\prime}$ forms a 
connected sum of $B$ and $B^{\prime}$, which reads, in our algebraic language,

$$
\begin{aligned}
B+B^{\prime} \stackrel{T_{r}+T_{l}^{\prime}=0}{=} B \# B^{\prime} & ={ }_{T_{l}}^{S}\left[T_{a}, T_{b}, T_{c}\right]_{T_{r}}^{S} \# \underset{-T_{r}}{S}\left[T_{a}^{\prime}, T_{b}^{\prime}, T_{c}^{\prime}\right]_{T_{r}^{\prime}}^{S} \\
& ={ }_{T_{l}}^{S}\left[\left(T_{a}, T_{b}, T_{c}\right)+\left(T_{a}^{\prime}, T_{b}^{\prime}, T_{c}^{\prime}\right)\right]_{T_{r}^{\prime}}^{S}={ }_{T_{l}}^{S}\left[T_{a}+T_{a}^{\prime}, T_{b}+T_{b}^{\prime}, T_{c}+T_{c}^{\prime}\right]_{T_{r}^{\prime}}^{S} .
\end{aligned}
$$

Before dealing with the other subcase, it is useful to prove a Lemma.

Lemma 3. A simultaneous rotation commutes with a connected sum. In algebraic words, this reads

$$
R_{n,-n}\left(B \# B^{\prime}\right)=R_{n,-n}(B) \# R_{n,-n}\left(B^{\prime}\right), n \in \mathbb{Z}
$$

Proof. Let $B={ }_{T_{l}}^{S}\left[T_{a}, T_{b}, T_{c}\right]_{T_{c}}^{S}$ and $B^{\prime}={ }_{-T_{c}}^{S}\left[T_{a}^{\prime}, T_{b}^{\prime}, T_{c}^{\prime}\right]_{T_{r}^{\prime}}^{S}$. Note that $B$ and $B^{\prime}$ have the same end-node state, and that the right external twist of $B, T_{c}$, cancels the left external twist of $B^{\prime},-T_{c}$, which is the most general situation in which a connected sum is viable. Then, we have

$$
\begin{aligned}
& R_{n,-n}(B) \# R_{n,-n}\left(B^{\prime}\right)=R_{n,-n}\left(\underset{T_{l}}{S}\left[T_{a}, T_{b}, T_{c}\right]_{T_{c}}^{S}\right) \# R_{n,-n}\left({ }_{-T_{c}}^{S}\left[T_{a}^{\prime}, T_{b}^{\prime}, T_{c}^{\prime}\right]_{T_{r}^{\prime}}^{S}\right) \\
& ={ }_{T_{l}+n}^{(-)^{n}}{ }_{n}\left[P_{n}^{S}\left(T_{a}, T_{b}, T_{c}\right)\right]_{T_{c}-n}^{(-)^{n} S} \#{ }_{-T_{c}+n}^{(-)^{n} S}\left[P_{n}^{S}\left(T_{a}^{\prime}, T_{b}^{\prime}, T_{c}^{\prime}\right)\right]_{T_{r}^{\prime}-n}^{(-)^{n} S} \\
& \stackrel{T_{c}-n+\left(-T_{c} n\right)=0}{=}=\stackrel{(-)^{n} S}{T_{l}+n}\left[P_{n}^{S}\left(T_{a}+T_{a}^{\prime}, T_{b}+T_{b}^{\prime}, T_{c}+T_{c}^{\prime}\right)\right]_{T_{r}^{\prime}-n}^{(-)^{n} S} \\
& =R_{n,-n}\left(\underset{T_{l}}{S}\left[T_{a}+T_{a}^{\prime}, T_{b}+T_{b}^{\prime}, T_{c}+T_{c}^{\prime}\right]_{T_{r}^{\prime}}^{S}\right) \\
& \stackrel{E q 9}{\Longrightarrow}=R_{n,-n}\left(B \# B^{\prime}\right),
\end{aligned}
$$

closing the proof.

If $T_{r}+T_{l}^{\prime}=2 n \neq 0$, we are in the second subcase. We can choose to do a rotation of $-\left(T_{r}+T_{l}^{\prime}\right)$ on either the right end-node of $B$ or on the left end-node of $B^{\prime}$ to annihilate the twist $T_{r}+T_{l}^{\prime}$ on the common edge. If we rotate the right end-node of $B$ by $-\left(T_{r}+T_{l}^{\prime}\right)$, we create crossings on $B$ and change $B^{\prime}$ s twists, but we want to stay with the trivial braid diagrams. To get around of this problem we simply need to perform a rotation of the opposite handedness on the left end-node of $B$ as well, such that $B$ is still in its trivial representation. In other words, we perform a simultaneous rotation of $T_{r}+T_{l}^{\prime}$ on $B$. Likewise, if we rotate the left end-node of $B^{\prime}$ to cancel the twist $T_{r}+T_{l}^{\prime}$, we should perform a simultaneous rotation of $-\left(T_{r}+T_{l}^{\prime}\right)$ on $B^{\prime}$. In either choice, we arrive at a situation legal for a connected sum. Nonetheless, each choice yields a result for $B+B^{\prime}$, a natural question is whether the two results "the same", or to be precise, equivalent? The answer is "Yes", which is not only true for this case but also valid for the case in Fig. 6(b).

We now prove this statement as a theorem. Here is the setup of the theorem. For two actively-interacting trivial braids $B$ and $B^{\prime}$, with $B$ adjacent to $B^{\prime}$ on the left, if the 
interaction condition holds, one may need to perform a simultaneous rotation on either $B$ or $B^{\prime}$ to remove the twist on their common edge for the interaction to happen by a connected sum.

Theorem 1. The resulting interaction $B+B^{\prime}$ between two actively interacting braids does not depend on the choice of the braid being simultaneously rotated.

Proof. Let $B={ }_{T_{l}}^{S}\left[T_{a}, T_{b}, T_{c}\right]_{T_{r}}^{S}$ and $B^{\prime}={ }_{T_{l}^{\prime}}^{ \pm}\left[T_{a}^{\prime}, T_{b}^{\prime}, T_{c}^{\prime}\right]_{T_{r}^{\prime}}^{ \pm S}$, which considers both cases in Fig. 6. $T_{r}+T_{l}^{\prime}=2 n, n \in \mathbb{Z}$ for $+S$, and $T_{r}+T_{l}^{\prime}=2 n+1, n \in \mathbb{Z}$ for $-S$. Then, $B+B^{\prime}=$ ${ }_{T_{l}}^{S}\left[T_{a}, T_{b}, T_{c}\right]_{T_{r}}^{S}+{ }_{T_{l}^{\prime}}^{ \pm S}\left[T_{a}^{\prime}, T_{b}^{\prime}, T_{c}^{\prime}\right]_{T_{r}^{\prime}}^{ \pm S}$, and we have

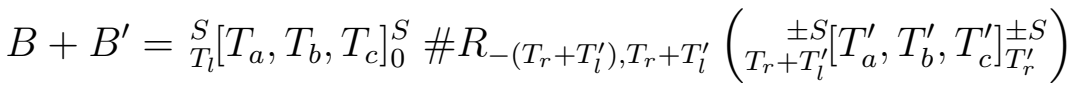

$$
\begin{aligned}
& ={ }_{T_{l}}^{S}\left[T_{a}, T_{b}, T_{c}\right]_{0}^{S} \# \stackrel{(-)^{T_{r}+T_{l}^{\prime}}( \pm S)}{\left({ }_{0}\right)}\left[P_{-\left(T_{r}+T_{l}^{\prime}\right)}^{ \pm S}\left(T_{a}^{\prime}, T_{b}^{\prime}, T_{c}^{\prime}\right)\right]_{T_{r}^{\prime}+T_{r}+T_{l}^{\prime}}^{\left(-T^{T_{r}+T_{l}^{\prime}}( \pm S)\right.} \\
& \stackrel{(-)^{T_{r}+T_{l}^{\prime}}( \pm S)=S}{\longrightarrow}={ }_{T_{l}}^{S}\left[T_{a}, T_{b}, T_{c}\right]_{0}^{S} \#{ }_{0}^{S}\left[P_{-\left(T_{r}+T_{l}^{\prime}\right)}^{ \pm S}\left(T_{a}^{\prime}, T_{b}^{\prime}, T_{c}^{\prime}\right)\right]_{T_{r}^{\prime}+T_{r}+T_{l}^{\prime}} \\
& ={ }_{T_{l}}^{S}\left[\left(T_{a}, T_{b}, T_{c}\right)+\left(P_{-\left(T_{r}+T_{l}^{\prime}\right)}^{ \pm S}\left(T_{a}^{\prime}, T_{b}^{\prime}, T_{c}^{\prime}\right)\right)\right]_{T_{r}^{\prime}+T_{r}+T_{l}^{\prime}}^{S}, \\
& B+B^{\prime}=R_{T_{r}+T_{l}^{\prime},-\left(T_{r}+T_{l}^{\prime}\right)}\left({ }_{T_{l}}^{S}\left[T_{a}, T_{b}, T_{c}\right]_{T_{r}+T_{l}^{\prime}}^{S}\right) \#{ }_{0}^{ \pm S}\left[T_{a}^{\prime}, T_{b}^{\prime}, T_{c}^{\prime}\right]_{T_{r}^{\prime}}^{ \pm S} \\
& ={ }_{T_{l}+T_{r}+T_{l}^{\prime}}^{\left(-T_{r}+T_{l}^{\prime}\right.}\left[P_{T_{r}+T_{l}^{\prime}}^{S}\left(T_{a}, T_{b}, T_{c}\right)\right]_{0}^{(-)^{T_{r}+T_{l}^{\prime} S}} \#{ }_{0}^{ \pm S}\left[T_{a}^{\prime}, T_{b}^{\prime}, T_{c}^{\prime}\right]_{T_{r}^{\prime}}^{ \pm S} \\
& \stackrel{(-)^{T_{r}+T_{l}^{\prime} S= \pm S}}{\longrightarrow}=\underset{T_{l}+T_{r}+T_{l}^{\prime}}{ \pm S}\left[\left(P_{T_{r}+T_{l}^{\prime}}^{S}\left(T_{a}, T_{b}, T_{c}\right)\right)+\left(T_{a}^{\prime}, T_{b}^{\prime}, T_{c}^{\prime}\right)\right]_{T_{r}^{\prime}}^{ \pm S},
\end{aligned}
$$

and

$$
\cong \begin{gathered}
{ }_{T_{l}}\left[\left(T_{a}, T_{b}, T_{c}\right)+\left(P_{-\left(T_{r}+T_{l}^{\prime}\right)}^{ \pm S}\left(T_{a}^{\prime}, T_{b}^{\prime}, T_{c}^{\prime}\right)\right)\right]_{T_{r}^{\prime}+T_{r}+T_{l}^{\prime}}^{S} \\
\underset{T_{l}+T_{r}+T_{l}^{\prime}}{ \pm S}\left[\left(P_{T_{r}+T_{l}^{\prime}}^{S}\left(T_{a}, T_{b}, T_{c}\right)\right)+\left(T_{a}^{\prime}, T_{b}^{\prime}, T_{c}^{\prime}\right)\right]_{T_{r}^{\prime}}^{ \pm S},
\end{gathered}
$$

where $\cong$ means "equivalent to". Note that $\pm S$ stands for the two cases respectively.

It is sufficient to prove Eq. 13. In view of Lemma 3, we may apply a simultaneous rotation of $T_{r}+T_{l}^{\prime}$ on the LHS of Eq. 13, but perform the rotation respectively on the two 
components of the connected sum and then take the connected sum. That is,

$$
\begin{aligned}
& \underset{T_{l}}{S}\left[\left(T_{a}, T_{b}, T_{c}\right)+\left(P_{-\left(T_{r}+T_{l}^{\prime}\right)}^{ \pm S}\left(T_{a}^{\prime}, T_{b}^{\prime}, T_{c}^{\prime}\right)\right)\right]_{T_{r}^{\prime}+T_{r}+T_{l}^{\prime}}^{S} \\
& \cong R_{T_{r}+T_{l}^{\prime},-\left(T_{r}+T_{l}^{\prime}\right)}\left({ }_{T_{l}}^{S}\left[T_{a}, T_{b}, T_{c}\right]_{0}^{S} \#{ }_{0}^{S}\left[P_{-\left(T_{r}+T_{l}^{\prime}\right)}^{ \pm S}\left(T_{a}^{\prime}, T_{b}^{\prime}, T_{c}^{\prime}\right)\right]_{T_{r}^{\prime}+T_{r}+T_{l}^{\prime}}^{S}\right) \\
& \stackrel{E q[10}{\Longrightarrow}=R_{T_{r}+T_{l}^{\prime},-\left(T_{r}+T_{l}^{\prime}\right)}\left(\stackrel{S}{T_{l}}\left[T_{a}, T_{b}, T_{c}\right]_{0}^{S}\right) \\
& \# R_{T_{r}+T_{l}^{\prime},-\left(T_{r}+T_{l}^{\prime}\right)}\left({ }_{0}^{S}\left[P_{-\left(T_{r}+T_{l}^{\prime}\right)}^{ \pm S}\left(T_{a}^{\prime}, T_{b}^{\prime}, T_{c}^{\prime}\right)\right]_{T_{r}^{\prime}+T_{r}+T_{l}^{\prime}}^{S}\right) \\
& =\stackrel{(-)^{T_{r}+T_{l}^{\prime}}{ }_{T_{l}+T_{r}+T_{l}^{\prime}}}{[}\left[P_{T_{r}+T_{l}^{\prime}}^{S}\left(T_{a}, T_{b}, T_{c}\right)\right]_{-\left(T_{r}+T_{l}^{\prime}\right)}^{\left(-T^{T_{r}+T_{l}^{\prime}}\right.} \\
& \# \stackrel{(-)^{T_{r}+T_{l}^{\prime} S}}{{ }_{T_{r}+T_{l}^{\prime}}^{\prime}}\left[P_{T_{r}+T_{l}^{\prime}}^{(-)^{T_{r}+T_{l}^{\prime}}{ }^{\prime}} P_{-\left(T_{r}+T_{l}^{\prime}\right)}^{ \pm S}\left(T_{a}^{\prime}, T_{b}^{\prime}, T_{c}^{\prime}\right)\right]_{T_{r}^{\prime}}^{(-)^{T_{r}+T_{l}^{\prime} S}} \\
& =\stackrel{(-)^{T_{r}+T_{l}^{\prime}} S}{T_{l}+T_{r}+T_{l}^{\prime}}\left[P_{T_{r}+T_{l}^{\prime}}^{S}\left(T_{a}, T_{b}, T_{c}\right)\right]_{-\left(T_{r}+T_{l}^{\prime}\right)}^{\left(-T^{T_{r}+T_{l}^{\prime}}\right.} \quad \# \underset{T_{r}+T_{l}^{\prime}}{ \pm S}\left[T_{a}^{\prime}, T_{b}^{\prime}, T_{c}^{\prime}\right]_{T_{r}^{\prime}}^{ \pm S} \\
& =\underset{T_{l}+T_{r}+T_{l}^{\prime}}{ \pm S}\left[P_{T_{r}+T_{l}^{\prime}}^{S}\left(T_{a}, T_{b}, T_{c}\right)\right]_{0}^{ \pm S} \# \underset{0}{ \pm S}\left[T_{a}^{\prime}, T_{b}^{\prime}, T_{c}^{\prime}\right]_{T_{r}^{\prime}}^{ \pm S} \\
& =\underset{T_{l}+T_{r}+T_{l}^{\prime}}{ \pm S}\left[\left(P_{T_{r}+T_{l}^{\prime}}^{S}\left(T_{a}, T_{b}, T_{c}\right)\right)+\left(T_{a}^{\prime}, T_{b}^{\prime}, T_{c}^{\prime}\right)\right]_{T_{r}^{\prime}}^{ \pm S},
\end{aligned}
$$

which is the very Eq. 13 . The second to the last line holds because the right subscript of the first term, $-\left(T_{r}+T_{l}^{\prime}\right)$, cancels the left subscript of the second term, $T_{r}+T_{l}^{\prime}$, and $(-)^{T_{r}+T_{l}^{\prime}}= \pm S$ respectively in the two cases. The third equality is valid for that

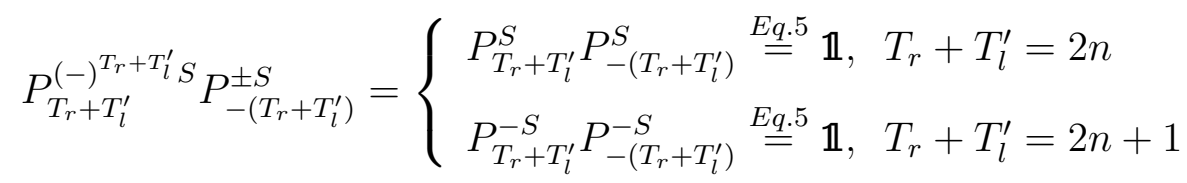

This completes the proof.
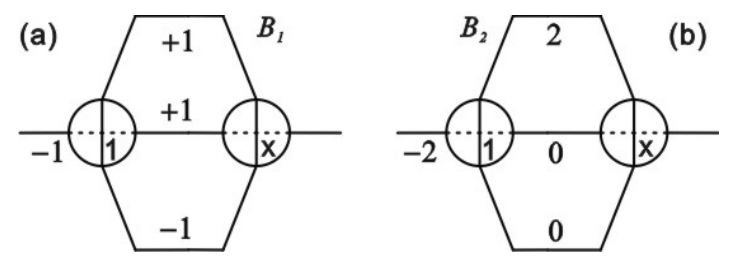

Figure 7: Two braids which interact actively.

Let us see an example. Fig. 7 shows two actively-interacting trivial braids[6], algebraically they are $B_{1}={ }_{-1}^{+}[1,1,-1]_{0}^{+}$and $B_{2}={ }_{-2}^{+}[T+2,0,0]_{0}^{+}$. One sees that

$$
\begin{aligned}
B_{1}+B_{2} & ={ }_{-1}^{+}[1,1,-1]_{0}^{+}+{ }_{-2}^{+}[2,0,0]_{0}^{+} \\
& ={ }_{-1}^{+}[1,1,-1]_{0}^{+} \# R_{2,-2}\left({ }_{-2}^{+}[2,0,0]_{0}^{+}\right) \\
& ={ }_{-1}^{+}[1,1,-1]_{0}^{+} \#{ }_{0}^{+}\left[P_{2}^{+}(2,0,0)\right]_{-2}^{+} \\
& ={ }_{-1}^{+}[1,1,-1]_{0}^{+} \#{ }_{0}^{+}[0,0,2]_{-2}^{+} \\
& ={ }_{-1}^{+}[1,1,1]_{-2}^{+} .
\end{aligned}
$$


On the other hand,

$$
\begin{aligned}
B_{1}+B_{2} & ={ }_{-1}^{+}[1,1,-1]_{0}^{+}+{ }_{-2}^{+}[2,0,0]_{0}^{+} \\
& =R_{-2,2}\left({ }_{-1}^{+}[1,1,-1]_{-2}^{+}\right) \#_{0}^{+}[2,0,0]_{0}^{+} \\
& ={ }_{-3}^{+}[-1,1,1]_{0}^{+} \#_{0}^{+}[2,0,0]_{0}^{+} \\
& ={ }_{-3}^{+}[1,1,1]_{0}^{+} .
\end{aligned}
$$

However, we have

$$
R_{2,-2}\left({ }_{-3}^{+}[1,1,1]_{0}^{+}\right)={ }_{-1}^{+}\left[P_{2}^{+}(1,1,1)\right]_{-2}^{+}={ }_{-1}^{+}[1,1,1]_{-2}^{+},
$$

satisfying Theorem 4 . Interestingly, $B_{2}+B_{1}$ does not work because while $B_{2}$ and $B_{1}$ have the same end-node state, the common twist -1 is an odd number, which violates the condition of interaction.

Equipped with this algebra, we shall prove our primary result:

Corollary 1. Given two actively interacting braids $B={ }_{T_{l}}^{S}\left[T_{a}, T_{b}, T_{c}\right]_{T_{r}}^{S}$ and $B^{\prime}={ }_{T_{l}^{\prime}}^{ \pm S}\left[T_{a}^{\prime}, T_{b}^{\prime}, T_{c}^{\prime}\right]_{T_{r}^{\prime}}^{ \pm S}$ satisfying the interaction conditions, the resulting braid $B^{f}=B+B^{\prime}$ conserves the quantities $T_{l}+T_{r}+T_{l}^{\prime}+T_{r}^{\prime}=T_{l}^{f}+T_{r}^{f}, \sum_{i=a}^{c}\left(T_{i}+T_{i}^{\prime}\right)=\sum_{i=a}^{c} T_{i}^{f}$ and $S^{2}$.

Proof. We immediately use the result from the proof of the previous theorem:

$$
B^{f}=\underset{T_{l}+T_{r}+T_{l}^{\prime}}{ \pm S}\left[\left(P_{T_{r}+T_{l}^{\prime}}^{S}\left(T_{a}, T_{b}, T_{c}\right)\right)+\left(T_{a}^{\prime}, T_{b}^{\prime}, T_{c}^{\prime}\right)\right]_{T_{r}^{\prime}}^{ \pm S}
$$

Because this result is independent of the form of the braids before the interaction according to the previous theorems, we need only examine the result of the interaction. $S^{2}$ is conserved as our final result shares the same value for $S^{2}$ of each of our initial states, and $S^{2}$ is invariant under the equivalence moves. We know that $T_{l}^{f}+T_{r}^{f}$ is conserved under the equivalence moves, and from our result we see that this conserved quantity is equal to $T_{l}+T_{r}+T_{l}^{\prime}+T_{r}^{\prime}$. Finally, $\sum_{i=a}^{c} T_{i}^{f}$ is the same regardless of the form of $P_{T_{r}+T_{l}^{\prime}}^{S}$, this immediately has the form of $\sum_{i=a}^{c}\left(T_{i}+T_{i}^{\prime}\right)$.

The three conserved quantities of this theorem have a clear meaning:

1. $T_{l}+T_{r}+T_{l}^{\prime}+T_{r}^{\prime}=T_{l}^{f}+T_{r}^{f}$, the total external twists before and after an interaction are the same;

2. $\sum_{i=a}^{c}\left(T_{i}+T_{i}^{\prime}\right)$, the total internal twists remains the same under interaction;

3. $S^{2}$, the interacting character of the braids is preserved. 


\section{Conclusions}

Conservation laws are a valuable tool in gaining understanding of the underlying structure of a theory. Elucidating the actual content of a theory and revealing information that could otherwise remain inaccessible. Equipped with invariants and conserved quantities, we are able to work to determine how the content of the theory relates to particle physics.

We have developed an algebraic notation for our braids, found a set of equivalence relations relating them, and developed conserved quantities associated with these relations. More importantly, we have found the relationship between these algebraic forms and the interaction of actively-interacting braids. From this we have found quantities conserved under interaction. These are dynamically conserved quantities, which are also conserved under the equivalence moves.

This allows us to work towards a better understanding of and a classification of the complete set of these braids. That we are able to show that actively-interacting braids interact with each other to produce actively-interacting braids allows us to generate an infinite set of such braids.

The next step in this work is to determine which of the conserved quantities found in this paper may be mapped to quantum numbers characterizing fundamental particles and to use the results we have found for interactions of braids to fully classify the set of braids.

\section{Acknowledgements}

The authors are indebted to their Advisor, Lee Smolin, for his encouragement and critical comments. We thank Sudance Bilson-Thompson for helpful discussions. Research at Perimeter Institute for Theoretical Physics is supported in part by the Government of Canada through NSERC and by the Province of Ontario through MRI.

\section{References}

[1] S. Bilson-Thompson, A topological model of composite preons, hep-ph/0503213.

[2] S. Bilson-Thompson, F. Markopoulou, L. Smolin, Quantum gravity and the standard model, Class. Quant. Grav., 24, 3975 (2007), hep-th/0603022

[3] S. Major and L. Smolin, Quantum deformation of quantum gravity, Nucl. Phys. B, 473, 267 (1996), gr-qc/9512020; R. Borissov, S. Major and L. Smolin, The geometry of quantum spin networks, Class. Quant. Grav., 13, 3183 (1996), gr-qc/9512043; L. Smolin, Quantum gravity with a positive cosmological constant, hep-th/0209079.

[4] J. Hackett, Locality and Translations in Braided Ribbon Networks, Class. Quant. Grav., 24, 5757 (2007), hep-th/0702198. 
[5] Y. Wan, On Braid Excitations in Quantum Gravity, arXiv:0710.1312.

[6] L. Smolin, Y. Wan, Propagation and Interaction of chiral states in quantum gravity, arXiv:0710.1548, Nucl. Phys. B, 796, 331 (2008).

[7] Carlo Rovelli, Loop Quantum Gravity, Living Rev.Rel. 1 (1998) 1, gr-qc/9710008; Quantum Gravity, Cambridge University Press, 2004.

[8] S. Bilson-Thompson, J. Hackett, and L. Kaufmann, 2009, in preparation.

[9] S. He, Y. Wan, Conserved Quantities and the algebra of Braid excitations in Quantum Gravity, Nucl. Phys. B 804, 286 (2008), arXiv:0805.0453.

[10] S. He, Y. Wan, C, P, and T of braid excitations in quantum gravity, Nucl. Phys. B 805, 1 (2008), arXiv:0805.1265.

[11] Y. Wan, Effective Theory of Braid Excitations of Quantum Geometry in terms of Feynman Diagrams, Nucl. Phys. B814:1-20 (2009), arXiv:0809.4464. 